\title{
Diagnóstico y tratamiento de la hernia obturatriz: Análisis de nuestra experiencia*
}

\author{
Drs. PABLO PRIEGO ${ }^{1}$, ANTONIO MENA², AGUSTÍN DE JUAN ${ }^{3}$
}

Servicio de Cirugía General y Digestivo. Hospital General de Castellón. Servicio de Cirugía General y Digestivo. Hospital Ramón y Cajal, Madrid.

Servicio de Cirugía General y Digestivo. Fundación Pública Hospital Comarcal Do Salnes, Pontevedra. España.

\section{Diagnosis and management of obturator hernia: analysis of our experience}

Background: Obturator hernia is a rare type of hernia. Because symptoms and signs are non-specific, diagnosis and treatment are often delayed, increasing the rate of strangulation and mortality. Material and Methods: A retrospective study was performed in 17 cases of obturator hernia at Ramón y Cajal Hospital between January 1986 and December of 2007. Results: All patients were women with a mean age of 77 years (range 19-88 years). Mean time from onset of symptoms to surgery was 3 days (range 0-10 days). Howship-Romberg sign was positive in five cases $(29,4 \%)$. Emergency surgery was performed in 16 cases $(94 \%)$ and elective surgery in one $(6 \%)$. CT has increased the rate of preoperative diagnosis from $16,6 \%$ to $41,2 \%$, however, the rate of strangulation of bowel was $47 \%$, requiring intestinal resection ten patients $(59 \%)$. Hernia repair was performed using polypropylene mesh in 8 cases $(47 \%)$ and by means of simple suture and apposition of the peritoneum in the rest 9 cases. Mean hospital postoperative stay was 11,65 days (range 4-26 days) and mortality was $23,5 \%$. Conclusion: Although CT scan has facilitated us the correct diagnosis of obturator hernia, decreasing the mean time from onset of symptoms to surgery to 3 days, we could not reduce the rate of intestinal resection and mortality.

Key words: Obturator hernia, Howship-Romberg sign, diagnosis, treatment, intestinal obstruction.

\section{Resumen}

La hernia obturatriz es una entidad rara, con frecuente ausencia de signos y síntomas específicos, lo que retrasa su diagnóstico y tratamiento, y por ello puede presentar una elevada tasa de estrangulación y mortalidad. Material y Métodos: Efectuamos un estudio retrospectivo sobre 17 casos de hernia obturatriz en el Hospital Ramón y Cajal entre enero de 1986 y diciembre de 2007. Resultados: Todos los pacientes eran mujeres con una edad media de 77 años (rango 19-88 años). El tiempo medio desde el inicio de los síntomas hasta la cirugía fue de 3 días (rango 1-10 días). El signo de Howship- Romberg fue positivo en 5 casos $(29,4 \%)$. Se efectuaron 16 intervenciones con carácter de urgente (94\%) y una de forma electiva (6\%).

\footnotetext{
*Recibido el 28 de Abril de 2009 y aceptado para publicación el 28 de Noviembre de 2009.

Correspondencia: Dr. Pablo Priego Jiménez

Calle de En Gran Avenida 3231312005 Castellón De La Plana, España.

E-mail: papriego@hotmail.com
} 
La realización de un TAC va a incrementar la tasa de diagnóstico preoperatorio de un $16,6 \%$ a un 41,2\%. La tasa de estrangulación fue de $47 \%$, requiriendo resección intestinal 10 pacientes (59\%). En 8 ocasiones se reparó el defecto herniario con una malla de polipropileno (47\%), siendo con cierre simple y aposición del peritoneo en los 9 restantes. La estancia media postoperatoria fue de 11,65 días (rango 4-26 días) y la tasa de mortalidad de 23,5\%. Conclusión: Aunque la realización del TAC ha incrementado la tasa de diagnóstico preoperatorio, disminuyendo el tiempo desde la aparición de los síntomas hasta la cirugía a 3 días, no hemos podido reducir la tasa de resección intestinal y mortalidad.

Palabras clave: Hernia obturatriz, signo de Howship-Romberg, diagnóstico, tratamiento, obstrucción intestinal.

\section{Introducción}

La hernia obturatriz, descrita por primera vez en 1724 por Ronsil, es una entidad rara, con una frecuencia de presentación entre un $0,05-0,14 \%$ de todas las hernias de la pared abdominal, y es causa de una obstrucción de intestino delgado entre un $0,2-1,6 \%$ de los casos $^{1-3}$.

En general, aparece en mujeres delgadas, ancianas y multíparas ${ }^{4-6}$. Debido a que los signos y síntomas son inespecíficos, el diagnóstico y el tratamiento a menudo se retrasa, siendo el diagnóstico preoperatorio únicamente posible en un 10-30\% de los $\operatorname{casos}^{6-10}$. Este retraso asocia un incremento de las tasas de estrangulación herniaria (que oscilan entre un $25-100 \%)^{1,5-7,10}$ y mortalidad $(12-70 \%)^{4-7,10,11}$.

En consecuencia, un diagnóstico y tratamiento precoces son esenciales para reducir la tasa de estrangulación y mortalidad.

El objetivo del estudio es analizar nuestra experiencia en el manejo de 17 casos de hernias obturatrices.

\section{Material y Método}

Se revisan de forma retrospectiva todos los casos de hernia obturatriz tratados en el Hospital Ramón y Cajal desde enero de 1986 hasta diciembre de 2007, analizándose las siguientes variables: sexo, edad, estado nutricional, enfermedades asociadas, clínica de presentación, pruebas complementarias, diagnóstico preoperatorio, tratamiento, morbilidad, mortalidad y evolución.

\section{Resultados}

Se identifican 17 casos de hernia obturatriz en 15 pacientes (cirugía urgente en 16 casos y electiva en 1 caso). Todos los pacientes eran mujeres con edad media de 77 años (rango 19-88 años). El tiempo medio desde el inicio de los síntomas hasta la cirugía fue de 3 días (rango 1-10 días). Los detalles de los pacientes aparecen reflejados en las Tablas 1 y 2 .
En el 50\% de los casos, los pacientes se encontraban en un estado de extrema delgadez y caquexia. La mayor parte (16 casos-94\%) presentaban signos y síntomas de obstrucción intestinal (dolor y distensión abdominal, náuseas, vómitos y ausencia de peristaltismo). El signo de Howship-Romberg (dolor en la cadera irradiado a la cara interna del muslo y rodilla ipsilaterales) fue positivo en 5 casos $(29,4 \%)$.

La radiología simple de abdomen reflejaba en todos los casos una dilatación importante del intestino delgado. Cuatro fueron estudiados mediante TAC (Figuras 1 y 2), demostrando una obstrucción intestinal secundaria a una hernia obturatriz incarcerada en todos los casos, y en dos pacientes se realizó una ecografía abdominal, que fue diagnóstica en uno de ellos. En total, se realiza un correcto diagnóstico preoperatorio de obstrucción intestinal secundaria a hernia obturatriz incarcerada en 7 casos $(41,2 \%)$.

Las vías de abordaje fueron: laparotomía media infraumbilical en 8 casos, laparotomía media suprainfraumbilical en 5 casos, inguinal en 3 casos y pararrectal derecha en una ocasión.

Los hallazgos intraoperatorios fueron: 8 casos de hernia estrangulada y perforada $(47 \%), 7$ casos de hernia incarcerada con intestino delgado viable (41\%), 1 caso de hernia obturatriz normal (cirugía electiva) y un caso de hernia obturatriz asociada a vólvulo de sigma (Figuras 3 y 4).

En todos los casos el órgano herniado fue el intestino delgado. En 11 ocasiones la hernia obturatriz fue derecha $(64,7 \%)$ y en seis izquierda. La tasa de estrangulación herniaria fue de $47 \%$, requiriendo resección intestinal 10 pacientes (59\%): 8 casos por presencia de una hernia estrangulada y perforada, y 2 casos debido a la perforación del intestino delgado durante la manipulación y reducción del saco herniario.

La hernia fue reparada usando una malla de polipropileno en 8 casos (47\%), siendo con cierre simple y aposición del peritoneo en los 9 restantes (53\%). La estancia media postoperatoria fue de 11,65 días (rango 4-26 días). Las complicaciones postoperatorias aparecen reflejadas en la Tabla 3.

Durante el seguimiento, un paciente presentó una suboclusión intestinal que se manejó de forma 
Tabla 1. Hernia obturatriz. Nuestra serie

\begin{tabular}{cccccccccc}
\hline Casos & Edad/Sexo & ASA & ECO/TC & SHR & $\begin{array}{c}\text { Diagnóstico } \\
\text { Preoperatorio }\end{array}$ & $\begin{array}{c}\text { Hallazgos } \\
\text { quirúrgicos }\end{array}$ & $\begin{array}{c}\text { Resección } \\
\text { intestinal }\end{array}$ & $\begin{array}{c}\text { Reparación } \\
\text { hernia }\end{array}$ & $\begin{array}{c}\text { Mortali- } \\
\text { dad }\end{array}$ \\
\hline 1 & $76 \mathrm{M}$ & IV & No & No & OIOD & HOEP & Sí & Malla & No \\
2 & $68 \mathrm{M}$ & III & No & SI & OIOD & HOI & No & Sutura directa & No \\
3 & $88 \mathrm{M}$ & III & No & No & OIOD & HOI & Sí & Sutura directa & No \\
4 & $77 \mathrm{M}$ & III & No & No & OIHI & HOI & No & Sutura directa & No \\
5 & $86 \mathrm{M}$ & III & No & SI & OIOD & HOEP & Sí & Sutura directa & No \\
6 & $86 \mathrm{M}$ & III & No & No & OIOD & HOEP & Sí & Sutura directa & No \\
7 & $81 \mathrm{M}$ & III & ECO & SI & OIHO & HOEP & Sí & Sutura directa & No \\
8 & $19 \mathrm{M}$ & I & No & No & HIR & HON & No & Malla & No \\
9 & $72 \mathrm{M}$ & IV & No & No & OIOD & HOEP & Sí & Malla & No \\
10 & $62 \mathrm{M}$ & III & ECO & SI & OIHO & HOI & No & Malla & Sí \\
11 & $79 \mathrm{M}$ & IV & No & No & OIOD & HOI vólvulo & No & Sutura directa & Sí \\
12 & $82 \mathrm{M}$ & IV & No & No & OIOD & HOEP & Sí & Sutura directa & No \\
13 & $84 \mathrm{M}$ & IV & TC & No & OIHO & HOI & No & Malla & No \\
14 & $86 \mathrm{M}$ & IV & No & SI & OIHO & HOI & Sí & Sutura directa & Sí \\
15 & $71 \mathrm{M}$ & III & TC & No & OIHO & HOEP & Sí & Malla & No \\
16 & $84 \mathrm{M}$ & III & TC & No & OIHO & HOI & No & Malla & No \\
17 & $79 \mathrm{M}$ & III & TC & No & OIHO & HOEP & Sí & Malla & Sí \\
\hline
\end{tabular}

M: Mujer. ASA: Sociedad Americana de Anestesiología. ECO: Ecografía abdominal. TC: Tomografía computarizada. SHR: Signo de Howship-Romberg. OIOD: Obstrucción intestinal de origen desconocido. OIHO: Obstrucción intestinal debido a hernia obturatriz. OIHI: Obstrucción intestinal debido a hernia inguinal. HIR: Hernia inguinal recidivada y no complicada. HOEP: Hernia obturatriz estrangulada y perforada. HOI: Hernia obturatriz incarcerada. HON: Hernia obturatriz normal.

Tabla 2. Enfermedades asociadas

\begin{tabular}{lcr}
\hline Enfermedades asociadas & \multicolumn{2}{c}{$\mathbf{n}$} \\
\hline $\begin{array}{l}\text { Cardíacas: Hipertensión, arritmias, } \\
\text { fallo cardíaco, otras }\end{array}$ & 7 pacientes & $(46,67 \%)$ \\
$\begin{array}{l}\text { Neurológicas: Parkinson, Alzheimer, } \\
\text { accidentes cerebro-vasculares }\end{array}$ & 5 pacientes & $(33,3 \%)$ \\
Artrosis y osteoporosis & & \\
$\begin{array}{l}\text { Enfermedad hepática: hepatitis, } \\
\text { cirrosis biliar primaria }\end{array}$ & 3 pacientes & $(20 \%)$ \\
$\begin{array}{l}\text { Embolismo pulmonar } \\
\begin{array}{l}\text { Cirugía abdominal previa: Histerecto- } \\
\text { mía, colecistectomía, hernia inguinal, } \\
\text { hernia obturatriz }\end{array}\end{array}$ & $\begin{array}{l}8 \text { pacientes } \\
(53,3 \%)\end{array}$ \\
\end{tabular}

Tabla 3. Complicaciones postoperatorias

\begin{tabular}{lc}
\hline $\begin{array}{l}\text { Complicaciones } \\
\text { postoperatorias }\end{array}$ & $\begin{array}{c}\text { n: } \mathbf{6} \text { pacientes } \\
(\mathbf{3 5 , 3 \% )}\end{array}$ \\
\hline Evisceración & 1 \\
Infección herida & 2 \\
Dehiscencia anastomosis & 2 \\
Infarto agudo de miocardio & 1 \\
Íleo paralítico & 1 \\
$\begin{array}{l}\text { Reintervención: } \\
\text { - Evisceración. }\end{array}$ & $2(11,7 \%)$ \\
- Dehiscencia anastomosis & \\
Mortalidad & $4(23,5 \%)$ \\
\hline
\end{tabular}



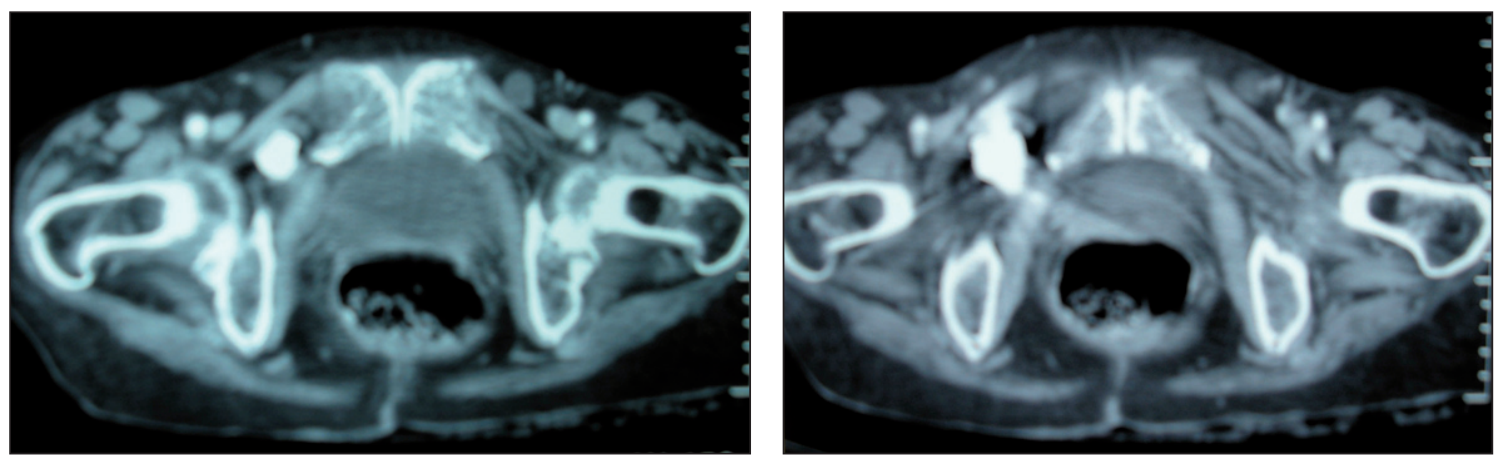

Figuras 1-2. TAC demuestra una hernia obturatriz derecha estrangulada con un segmento de intestino delgado entre el músculo pectíneo y el obturador externo.

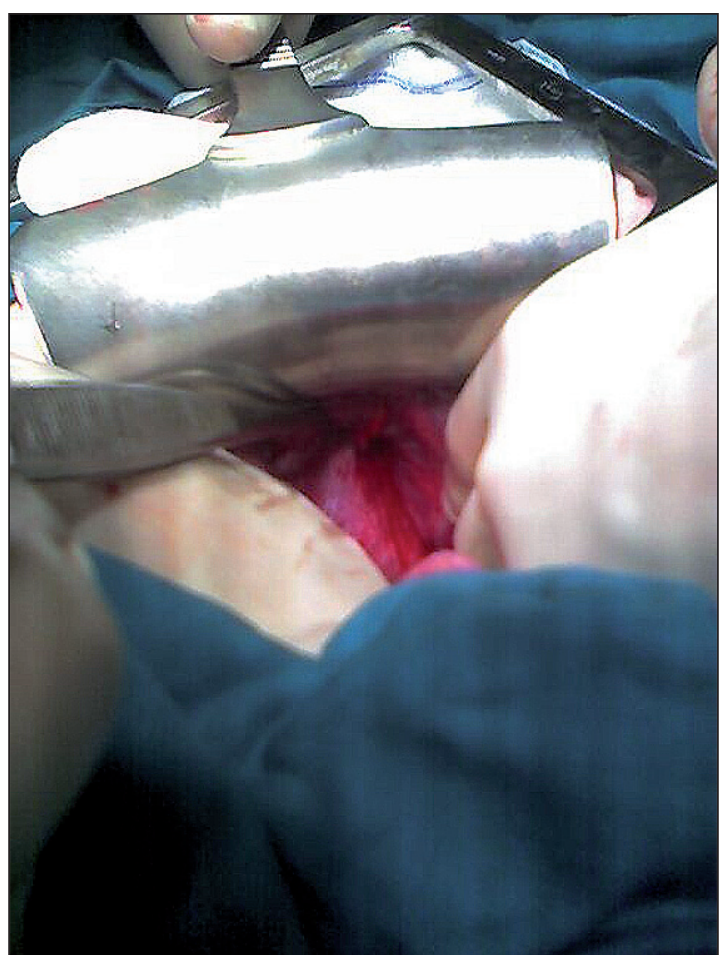

Figura 3. Orificio obturador.

conservadora, otro desarrolló una fístula cutánea (paciente que había sufrido evisceración), uno de los pacientes presentó una recurrencia de la hernia obturatriz 13 años después de la primera reparación (herniorrafía) y otro paciente fue operado de una hernia obturatriz izquierda estrangulada y 3 años después de otra en el lado contralateral (Tabla 4).

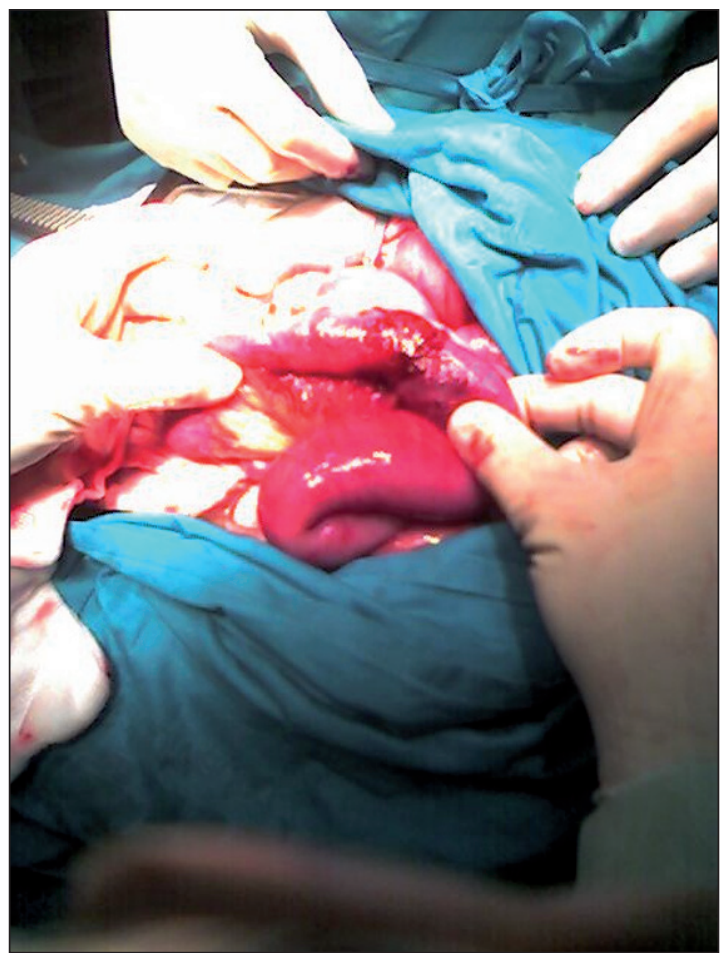

Figura 4. Intestino delgado estrangulado.

\section{Discusión}

La hernia obturatriz es una entidad rara, que en general, aparece en mujeres delgadas, ancianas y multíparas, y con una frecuencia de presentación entre un $0,05-0,14 \%$ de todas las hernias de la pared abdominal ${ }^{1-6}$. 
Tabla 4. Comparación de los resultados en el tratamiento de la hernia obturatriz en diferentes series

\begin{tabular}{lccccccc}
\hline Autor-año & $\mathbf{n}$ & $\begin{array}{c}\text { Diagnóstico } \\
\text { preoperatorio } \\
(\%)\end{array}$ & $\begin{array}{c}\text { Tiempo } \\
\text { hasta } \\
\text { cirugía } \\
\text { (días) }\end{array}$ & $\begin{array}{c}\text { Tasa } \\
\text { estrangu- } \\
\text { lación (\%) }\end{array}$ & $\begin{array}{c}\text { Resección } \\
\text { intestinal } \\
(\%)\end{array}$ & $\begin{array}{c}\text { Morbilidad } \\
(\%)\end{array}$ & $\begin{array}{c}\text { Mortalidad } \\
(\%)\end{array}$ \\
\hline Yokohama 1999 & 36 & 58,3 & 5,5 & ND & 58,3 & 22,2 & 11,1 \\
Mena 2002 & 12 & 16,6 & 3,6 & 50 & 58,3 & 25 & 16,6 \\
Nakayama 2002 & 12 & 66,6 & 9 & ND & 41,6 & 16,6 & 8,3 \\
Hennekinne-Mucci 2003 & 17 & 23,5 & 5,3 & 94,1 & 64,7 & 52,9 & 35,3 \\
Kammori 2004 & 43 & 60,4 & 4,4 & ND & 37,2 & 86 & 18,6 \\
Chang 2005 & 6 & 50 & 5,2 & 100 & 100 & 16,6 & 16,6 \\
Thanapaisan 2006 & 61 & 36,07 & 3,13 & 67,21 & 65,57 & 13,1 & 11,5 \\
Rodríguez 2008 & 16 & 18,8 & 5,9 & 75 & 75 & 75 & 18,8 \\
Priego 2009* & 17 & 41,2 & 3 & 47 & 59 & 35,3 & 23,5 \\
\hline
\end{tabular}

ND no disponible. *Nuestra serie reflejada en este artículo.

Modificado de Rodríguez JI, Codina A, Maroto A, et al. Obturator hernia: clinical analysis of 16 cases and algorithm for its diagnosis and treatment. Hernia 2008; 12: 289-297.

La etiología y patogénesis de la hernia obturatriz se debe a una disminución del tejido graso y linfático preperitoneal que ocupa el canal obturador. Aquellas situaciones que incrementan la presión abdominal, como la delgadez extrema, caquexia, embarazo y multiparidad, EPOC, estreñimiento crónico, cifoescoliosis, ascitis, etc, van a ser factores predisponentes ${ }^{3,5,6,10,12,13}$.

Son más frecuentes en el lado derecho $(64,7 \%$ en nuestra serie), debido a que el colon izquierdo cubre el agujero obturador izquierdo $0^{3,4,6,7,11}$, y en un $50-60 \%$ de los casos son hernias tipo Richter $4,6,7,10,11$.

Normalmente el saco contiene intestino delgado (íleon) (100\% en nuestra serie), pero también se ha descrito la presencia del colon, apéndice, ovario, trompa de Falopio, epiplón y vejiga ${ }^{3,6,7,10,14}$.

Los síntomas son inespecíficos, debutando en un $90 \%$ de los pacientes como un cuadro de obstrucción intestinal $1^{1,6-8}$. En estos pacientes, la presencia de una masa en el tacto rectal o vaginal, nos debe hacer sospechar su existencia. El signo de Howship-Romberg

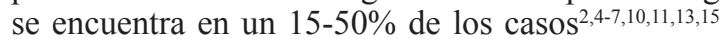
( $29,4 \%$ en nuestra serie), pero no es patognomónico, y a menudo se confunde con un proceso osteoarticular $(26,7 \%$ de nuestros pacientes presentaban una artrosis degenerativa y/o osteoporosis).

La inespecificidad de sus síntomas hace que un correcto diagnóstico preoperatorio sólo sea posible entre un $10-30 \%$ de los casos (aunque en las series de Yokohama, Nakayama, Chang y Kammori se llega a un $50-60 \%)^{3-6,10,11,15,16}$. En nuestro estudio, la tasa de diagnóstico preoperatorio es de $41,2 \%$. En este punto creemos que es importante la realización de un TAC abdominopélvico, que en nuestra experiencia ha conseguido incrementar la tasa de diagnóstico preoperatorio desde un $16,6 \%$ en el año 2002 hasta el 41,2\% actual.

Aunque Cubillo ${ }^{17}$, en 1983, fue el primero en describir la utilidad del TAC abdominopélvico en el diagnóstico de hernias obturatrices, en nuestro hospital, éste no fue utilizado en el diagnóstico de esta patología hasta el año 2003. Sin embargo, al igual que refiere Yokohama ${ }^{4}$, a pesar de que el TAC nos ha facilitado un correcto diagnóstico de la hernia obturatriz, disminuyendo el tiempo desde el inicio de los síntomas hasta la cirugía de 3,6 a 3 días, no hemos podido reducir la tasa de resección intestinal ni de mortalidad. A pesar de esto, recomendamos que en caso de mujeres multíparas y de edad avanzada con sintomatología de obstrucción intestinal, se debería realizar un TAC abdominopélvico lo más pronto posible. Esta recomendación confronta con la opinión de otros autores, que abogan por una laparotomía precoz: "La hernia obturatriz necesita una laparotomía, no un diagnóstico"2. Sin embargo, la inespecificidad de los signos y síntomas en estos pacientes, asociados a que en general son personas de edad avanzada y con un deteriorado estado de salud, hace que prefiramos realizar de forma precoz un TAC, para asegurar el diagnóstico antes de la cirugía.

Se ha descrito una gran variedad de abordajes 
quirúrgicos sin que exista una técnica estandari$\mathrm{zada}^{1,7,18,19}$. En nuestra experiencia, siempre que tengamos un diagnóstico preoperatorio de hernia obturatriz o sospecha de sufrimiento intestinal, la elección sería una laparotomía media exploradora. Carecemos de experiencia en el abordaje laparoscópico en este tipo de patología $a^{5,12,20-22}$.

En general, se considera suficiente un cierre primario del orificio ${ }^{10,11}$ en el caso de hernias de pequeño tamaño, sin embargo, en grandes defectos herniarios no siempre es posible dada la pobre distensión de los tejidos adyacentes. Algunos autores utilizan flaps de fascia peritoneal, pared de vejiga o fundus uterino, sin embargo, la tendencia actual es la reparación del defecto usando material protésico ${ }^{3-8}$ cubriendo correctamente la malla con peritoneo. La tasa de recurrencia se encuentra en torno al $10 \%{ }^{6-8}$, en nuestra serie $6,7 \%$.

El tiempo medio entre el inicio de los síntomas y el tratamiento oscila en la literatura en 4-6 días ${ }^{5-7}$. En nuestra serie fue de 3 días. Dado que es muy difícil acortar el tiempo desde el inicio de los síntomas hasta la consulta hospitalaria, es esencial una sospecha clínica y un tratamiento quirúrgico precoz desde la llegada del paciente al hospital.

Nuestra tasa de mortalidad fue de $23,5 \%$, y se encuentra en el rango descrito en la literatura (12$70 \%)^{1,5,7,10,11,13}$, debiéndose en su mayor parte a la edad avanzada de los pacientes, el retraso en el diagnóstico, la gangrena del intestino y el pobre estado nutricional y las enfermedades de base del paciente.

En conclusión, un diagnóstico y tratamiento precoz es esencial para reducir la tasa de estrangulación $(25-100 \%)^{1,3-7,23,24}$ y mortalidad. Por este motivo, debemos sospecharlo en mujeres ancianas, delgadas y multíparas con síntomas de obstrucción intestinal, especialmente en ausencia de cirugías abdominales previas, y debemos dirigir la exploración hacia la realización de un tacto rectal y vaginal, así como de un TAC abdominopélvico lo más pronto posible, con la idea de no demorar la intervención quirúrgica en el caso de que se efectúe un diagnóstico de hernia obturatriz incarcerada.

\section{Referencias}

1. Lo CY, Lorente TG, Lau PW. Obturador hernia presenting as small bowel obstruction. Am J Surg 1994; 167 : 396-398.

2. Ziegler DW, Rhoads JE Jr. Obturador hernia needs a laparotomy, not a diagnosis. Am J Surg 1995; 170: 67 68.

3. Nakayama T, Kobayashi S, Shiraishi K, Nishiumi T, Mori S, Isobe K, et al. Diagnosis and treatment of obturator hernia. Keio J Med 2002; 51: 129-132.
4. Yokoyama Y, Yamaguchi A, Isogai M, Hori A, Kaneoka Y. Thirty-six cases of obturador hernia: does computed tomography contribute to postoperative outcome? World J Surg 1999; 23: 214-217.

5. Chang SS, ShanYS, Lin YJ, Tai YS, Lin PW. A review of obturator hernia and a proposed algorithm for its diagnosis and treatment. World J Surg 2005; 29: 450-454.

6. Rodríguez JI, Codina A, Maroto A, Puig J, Sirvent JM, Garsot E, et al. Obturador hernia: clinical analysis of 16 cases and algorithm for its diagnosis and treatment. Hernia 2008; 12: 289-297.

7. Mena A, De Juan A, Larrañaga I, Aguilera A, Fernández de Bobadilla L, Fresneda V. Hernia obturatriz: análisis de nuestra serie y revisión de la enfermedad. Cir Esp 2002; 72: 67-71.

8. Calvo AM, Herrera J, Iñigo JJ, Zazpe C, Ariceta J, Lera JM. Hernia obturatriz diagnosticada preoperatoriamente como causa de obstrucción intestinal. Cir Esp 1999; 65: 437-439.

9. García A, Gómez J, González L, Sánchez J, Pina J, Cuadrado F y cols. Diagnóstico preoperatorio de hernia obturatriz por radiología simple. Cir Esp 2000; 67: 216217.

10. Thanapaisan C, Thanapaisal C. Sixty-one cases of obturator hernia in Chiangrai Regional Hospital: Retrospective study. J Med Assoc Thai 2006; 89: 2081-2086.

11. Kammori M, Mafune K, Hirashima T, Kawahara M, Hashimoto M, Ogawa T, et al. Forty-three cases of obturator hernia. The American Journal of Surgery 2004; 187: 549-552.

12. Shapiro K, Patel S, Choy C, Chaudry G, Khalil S, Ferzli G. Totally extraperitoneal repair of obturator hernia. Surg Endosc 2004; 18: 954-956.

13. Hennekinne-Mucci S, Pessaux P, Du Plessis D, Regenet N, Lermite E, Arnaud JP. Hernie obturatrice étranglée: à propos de 17 cases. Annales de Chirurgie 2003; 128 : 159-162.

14. Alexandre JH, Bouillot JL, Dehni N (1994). Traitement chirurgical des hernies obturatrices. Encycl Méd Chir (Elsevier, Paris-France), Techniques chirurgicalesAppareil digestif , 40-155, 6p.

15. Hsu CH, Wang CC, Jeng LBJ, Chen MF. Obturator hernia: a report of eight cases. Am Surg 1993; 59: 709711.

16. Nishina M, Fujii C, Ogino R, Kobayashi R, Kumada K, Yamane K, et al. Preoperative diagnosis of obturador hernia by computed tomography. Semin Ultrasound CT MR 2002; 23: 193-196.

17. Cubillo E. Obturator hernia diagnosed by computed tomography. Am J Roentgenol 1983; 140: 735-736.

18. Bergstein JM, Condon RE. Obturator hernia: current diagnosis and treatment. Surgery 1996; 119: 133-136.

19. Shandalakis LJ, Androulakis J, Colborn GL, Shandalakis JE. Obturator hernia. Embriology, anatomy and surgical applications. Surg Clin North Am 2000; 80: 71-84. 
20. Crescienzo D, Faranda C, Perrot L, Champault G. Laparoscopic treatment of a strangulated obturador hernia. Hernia 1998; 2: 203-205.

21. Yau KK, Siu WT, Chau CH, Yang PC, Li MK. Laparoscopic management of incarcerated obturator hernia. Can J Surg 2005; 48: 76-77.

22. Perry CP, Hantes JM. Diagnosis and laparoscopic repair of type I obturator hernia in women with chronic neuralgic pain. JSLS 2005; 9: 1338-1341.

23. Bueno J, Serralta A, Dobón F, Galeano J, Ballester C, Ibáñez F y cols. Reparación de hernia obturatriz con malla de polipropileno. Cir Esp 2000; 67: 516-517.

24. Rimmer JA, Wharton S. Bilateral and recurrent obturador hernia. Br J Clin Pract 1990; 44: 784. 